\title{
A Study of Association between Preoperative Fine Needle Aspiration Cytology and Post-Operative Histopathological Examination of Solitary Thyroid Nodule among Patients Attending North Bengal Medical College and Hospital, Siliguri
}

\author{
Prasanta Kumar Gure¹, Dhrupad Ray², Radheshyam Mahato³, Manotosh Dutta ${ }^{4}$ \\ Soma Mandal ${ }^{5}$, Arunava Ghosh ${ }^{6}$, Arijit Das ${ }^{7}$
}

\begin{abstract}
${ }^{1}$ Department of ENT, North Bengal Medical College and Hospital, Siliguri, West Bengal, India. ${ }^{2}$ Department of ENT, North Bengal Medical College and Hospital, Siliguri, West Bengal, India. ${ }^{3}$ Department of ENT, North Bengal Medical College and Hospital, Siliguri, West Bengal, India. ${ }^{4}$ Department of ENT, North Bengal Medical College and Hospital, Siliguri, West Bengal, India. 5Department of ENT, North Bengal Medical College and Hospital, Siliguri, West Bengal, India. ${ }^{6}$ Department of ENT, North Bengal Medical College and Hospital, Siliguri, West Bengal, India. ${ }^{7}$ Department of ENT, North Bengal Medical College and Hospital, Siliguri, West Bengal, India.
\end{abstract}

\section{ABSTRACT}

\section{BACKGROUND}

FNAC (Fine Needle Aspiration Cytology) is an essential tool in the preoperative diagnosis and preoperative planning for surgery of solitary thyroid nodules. It is a cost effective procedure that provides specific diagnosis rapidly with minimal complication. The purpose of the study was to evaluate the accuracy of Fine Needle Aspiration Cytology as a diagnostic tool in detecting malignancy in case of solitary thyroid nodule by correlating the preoperative fine needle aspiration cytology (USG Guided) findings with post-operative histopathology findings of the excised specimen in a sub Himalayan tertiary medical college \& hospital.

\section{METHODS}

It was a cross sectional hospital based study conducted on 30 patients in the period from April 2015 to March 2016 after acceptance from West Bengal University of Health Sciences and approval from ethical committee. All admitted patients having solitary thyroid nodule (confirmed clinically \& ultrasonographically), biochemically euthyroid and giving consent for surgery were included in this study. Patients who are medically unfit for surgery, biochemically hypo- or hyper-thyroid and not giving consent for surgery were excluded from the study.

\section{RESULTS}

The sensitivity, specificity, accuracy, positive predictive value and negative predictive value of FNAC for diagnosis of neoplastic solitary thyroid nodule were $75 \%, 94.44 \%, 86.67 \%, 90 \%$ and $85 \%$. Commonest malignancy detected was papillary carcinoma in 9 patients.

\section{CONCLUSIONS}

It is evident from our study that USG guided FNAC should be regarded as the investigation of choice and first line investigation in evaluation of cases with solitary thyroid nodule due to its simplicity, high diagnostic accuracy, easy to perform procedure and absence of significant complications.
Corresponding Author:

Dr. Dhrupad Ray,

Flat No. 2C, Woodland Park Residency, P. S-M-Atigara Dist., Darjeeling-734012, West Bengal, India.

E-mail: dhrupadray@gmail.com

DOI: $10.14260 / j e m d s / 2020 / 404$

Financial or Other Competing Interests: None.

How to Cite This Article:

Gure PK, Ray D, Mahato R, et al. A study of association between preoperative fine needle aspiration cytology and postoperative histopathological examination of solitary thyroid nodule among patients attending north Bengal medical college and hospital, Siliguri. J. Evolution Med. Dent. Sci. 2020;9(25):1854-1858, DOI: 10.14260/jemds/2020/404

Submission 02-01-2020,

Peer Review 25-05-2020,

Acceptance 01-06-2020,

Published 22-06-2020.

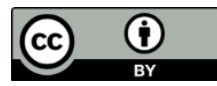

\section{KEY WORDS}

Solitary Thyroid Nodule, FNAC 


\section{BACKGROUND}

The thyroid gland is a prime endocrine gland of the body. It is unique among the endocrine organs in that it is the largest endocrine gland of the body and first to develop in the foetus. The normal thyroid gland is impalpable. The term 'goitre' is used to describe generalised swelling of the thyroid gland. It may be of two types: diffuse and nodular. Nodular goitre, the most common lesion of the thyroid gland, is of two types: solitary and multi- nodular goitre. A solitary thyroid nodule is a discrete swelling within the thyroid gland that is palpably and/or ultrasonographically distinct from the surrounding thyroid parenchyma. ${ }^{1}$

On investigation, many apparently solitary nodules are found to be a part of multinodular goitre. The term 'dominant' is used for similar swelling in a gland with clinical evidence of generalised abnormality in the form of a palpable contra lateral lobe or generalised mild nodularity. Nodular goitre is more common in iodine deficient areas like sub-Himalayan belt and is more common in middle aged females. The prevalence of thyroid swelling ranges from $4-10 \%$ in general adult population and from $0.2 \%$ to $1.2 \%$ in children. ${ }^{2}$

The majority of clinically diagnosed thyroid nodules are non-neoplastic; only 5-30\% are malignant and requires surgical intervention. ${ }^{3}$

A truly solitary thyroid nodule in a patient under 20 years old has a $25 \%$ chance of malignancy. The risk lessens in middle age to around $7-10 \%$ and increases again after the age of 50 when in men the likelihood of a solitary thyroid mass being malignant is $20-40 \% .{ }^{4}$ The main aim of evaluating a thyroid nodule is to detect any malignant transformation so that safe and effective surgery can be offered at the earliest to those who actually need it. The other patients can be managed with a conservative approach and meticulous follow up, so that indiscriminate operation can be avoided.

A variety of diagnostic tests like ultrasonography, Fine Needle Aspiration Cytology, thyroid nuclear scan and many more are available for evaluation of goitre. FNAC is widely accepted as the most cost-effective diagnostic procedure in the assessment of thyroid nodules and also helps in selecting patients preoperatively for surgery. ${ }^{5}$ It is a simple, less expensive, readily available and quick to perform procedure with excellent patient compliance \& superior diagnostic reliability. FNAC is however not without limitations; accuracy is lower in suspicious cytology and in follicular neoplasm. Limitations of FNAC are related to

- Specimen adequacy.

- Sampling techniques.

- Skill of performing aspiration.

- Interpretation of the aspirate.

- Overlapping cytological features between benign and malignant follicular neoplasm.

- In detection of papillary carcinoma with associated thyroid pathology like multinodular goitre, thyrotoxicosis and cystic changes. ${ }^{6}$

Ultrasonography (USG) is very helpful in detecting size of the tumour, diagnosing multinodular goitre, excluding contralateral lobe involvement and determining the nature of the solitary nodule as solid or cystic. Use of ultrasonographic guidance in performing FNAC increases significantly the sensitivity, specificity and accuracy of the procedure as compared to conventional palpation guided FNAC. ${ }^{7}$

The histopathological examination of the post-operative excised thyroid specimen is considered the final diagnostic test in spite of various nonsurgical and non-invasive investigations.

This study attempts to correlate pre-operative USG guided FNAC with post-operative histopathological examination in case of solitary thyroid nodule.

\section{METHODS}

This was a cross sectional hospital based study conducted on 30 indoor patients fulfilling the inclusion and exclusion criteria in the period from April 2015 to March 2016 in the department of ENT and Head \& Neck surgery of North Bengal Medical College and Hospital, a sub Himalayan tertiary care hospital after proper clearance from ethical committee. Sample size was calculated using the Epi-info ${ }^{\mathrm{TM}}$ software using local prevalence data.

The inclusion criteria included patients presenting with solitary thyroid nodule (diagnosed clinically and by ultrasonography) irrespective of age and sex and are being planned for surgery, having Normal thyroid hormone profile, Preoperative USG guided FNAC from the nodule done and Pre anaesthetic fitness done. The exclusion criteria being all patients with co morbidities or unfit for surgery, patients refusing surgery and not giving consent for the study.

The patients attending at OPD of Otorhinolaryngology and Head \& Neck Surgery at North Bengal Medical College and Hospital with thyroid swelling were examined clinically after taking detailed history and Local examination of thyroid. Preoperative indirect laryngoscopy was done in all cases Those patients diagnosed clinically to have solitary thyroid nodule were further investigated by Ultrasonography (USG) for confirmation of diagnosis along with USG guided FNAC for cytological diagnosis of the aspirate. Routinely, thyroid status was determined by estimation of serum T3, T4, TSH in all patients. The euthyroid patients who were planned for thyroid surgery were then admitted after preanaesthetic fitness done. The consenters were then examined clinically with detailed history as per pretested, semi structured questionnaires.

The postoperative specimen was sent to college pathology for histopathological examination. Follow up was done routinely for every patient after 6,12 and 24 weeks and in between when required. We also followed up all the subjects for further intervention as an ethical responsibility.

\section{Statistical Analysis}

Sample size was calculated using the Epi-info ${ }^{\mathrm{TM}}$ software using local prevalence data. Chi-square analysis were applied for statistical analysis; done using SPSS software. 


\section{RESULTS}

A total of 30 patients with solitary nodule were studied. As shown in table 1, 25 (83.3\%) were female and 5 (16.6\%) were male. The age of the patients ranged from 18 to 60 years. The mean age of presentation in female was 33.32 and in male were 45.8 with a standard deviation of 11.82 and 10.11 respectively. In our study, out of 30 patients, 19 patients $(63.33 \%)$ were from Hindu religion and 11 patients (36.66\%) were from Muslim religion. Most of the patients (86.8\%) presented with neck swelling.

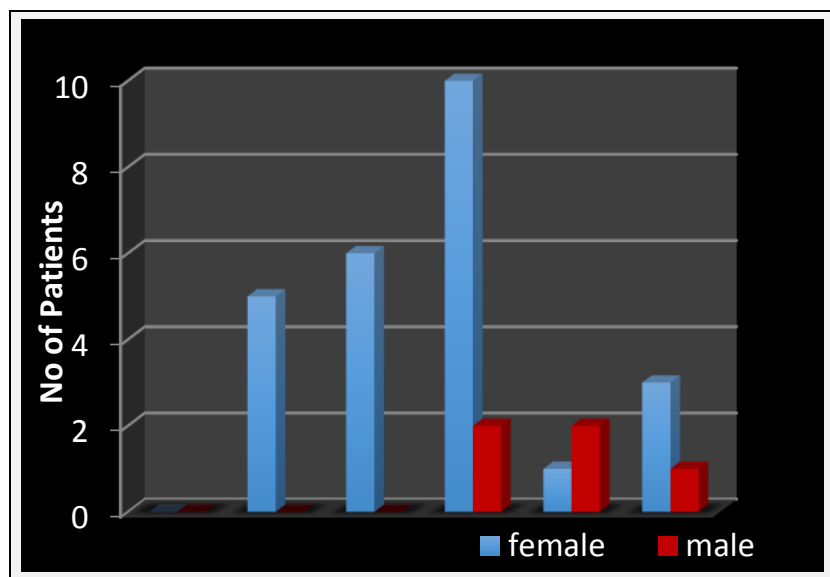

Figure 1. Distribution of Study Subjects according to Age and $\operatorname{Sex}(n=30)$

The residence of our study population involves the sub Himalayan districts including Dooars region. In cases of benign lesions, both Darjeeling and Coochbehar districts individually represent same of $27.77 \%$ of affected population. In malignant cases, Jalpaiguri represents $33.33 \%$ of affected populations followed by Darjeeling. No case of malignancy was found from patients of Coochbehar district.

We did USG guided FNAC in all the cases. First we detected the nature of the solitary thyroid nodule, whether it is solid or cystic. Out of 30 cases of solitary thyroid nodule 17 nodules were cystic where as 11 nodules were solid in consistency. 2 cases of papillary carcinoma show cystic change in solid thyroid nodule.

Histopathological examination of the operated specimen was done for all the patients in our study the result of which shown benign condition in 18 patients and malignancy in 12 patients. Out of 18 benign cases diagnosed by histopathology, 14 cases $(77.78 \%$ of benign cases) were found to have colloid nodule whereas papillary CA accounted for $75 \%$ of malignant cases (total 9 cases).

The aim of our study was to compare the preoperative USG guided FNAC with the postoperative histopathology report and correlate between the two. We have found the following discrepancies in this regard:

a. 1 case of follicular neoplasm diagnosed on FNAC was found to be a case of Hashimoto's thyroiditis on HPE.

b. Out of 17 patients diagnosed as colloid goitre on USG guided FNAC, 3 were found to have papillary carcinoma on HPE.

c. 1 case of papillary CA on FNAC was found to be follicular variant of papillary CA.

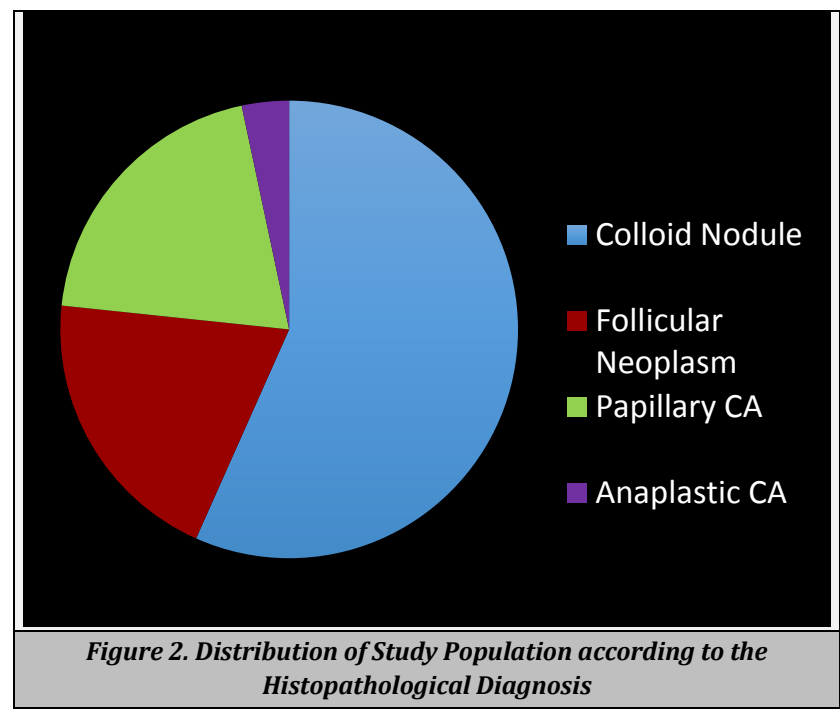

\begin{tabular}{|c|c|c|c|c|c|c|c|}
\hline \multirow[b]{3}{*}{ FNAC } & \multirow[b]{3}{*}{ Total } & \multicolumn{6}{|c|}{ Histopathology } \\
\hline & & \multicolumn{2}{|c|}{ Malignant } & \multicolumn{4}{|c|}{ Benign } \\
\hline & & $\underset{\frac{\pi}{3}}{\frac{7}{\pi}}$ & 竎 & 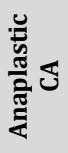 & 름 & 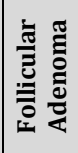 & 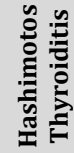 \\
\hline $\begin{array}{c}\text { Malignant } \\
\text { Papillary CA } \\
\text { Anaplastic CA }\end{array}$ & $\begin{array}{l}6 \\
1\end{array}$ & 6 & 0 & 1 & & & \\
\hline \begin{tabular}{|c|} 
Benign \\
Colloid Nodule \\
Follicular Neoplasm \\
Hashimoto's Thyroiditis \\
\end{tabular} & $\begin{array}{l}17 \\
6 \\
\end{array}$ & 3 & 2 & & 14 & 3 & 1 \\
\hline
\end{tabular}

So, out of 30 cases, USG guided FNAC has correctly diagnosed 26 cases.

So, in our study true positive, true negative, false positive and false negative cases are 9, 17, 1 and 3 respectively. Statistical analysis revealed Sensitivity of $75 \%$, Specificity of $94.44 \%$, Accuracy (effectiveness) of $86.67 \%$, Positive predictive value of $90 \%$ and Negative predictive value of $85 \%$. As follicular neoplasm, shown on FNAC, may be a case of follicular adenoma and carcinoma that can only be differentiated by histopathology, so the follicular neoplasm on FNAC that came to be follicular carcinoma HPE has been regarded as true positive and the follicular neoplasm on FNAC that came to be follicular adenoma on HPE has been regarded as true negative. Among 25 female patients 9 cases were found to be malignant whereas 3 out of 5 male patients were found to have malignancy. So $36 \%$ of female and $60 \%$ of male were found to have malignancy. The overall malignancy among all cases is $40 \%$.

So, in our study, most of the patients with solitary thyroid nodule were females but as far as malignancy is concerned it were the male patients who have the higher incidence.

\section{DISCUSSION}

In our study we have taken 30 patients with solitary thyroid nodules which were diagnosed preoperatively with USG guided FNAC. 
We have considered the patients of all age and sex. In our study the age of the patients was between 18-60 years. According to Ramchandraiah $\mathrm{C}$ et al in a study at Hyderabad showed that the mean age of the patients with solitary nodule was 44 and mean age of malignant cases were 36 . Out of 237 cases $83.9 \%$ were females and $16 \%$ were males and most patients were in the age group of 20-39 years. The female to male ratio was $5.2: 1{ }^{8}$ In our study we also found that 15 patients $(50 \%)$ were in the age group of $20-40$ years and $83.3 \%$ patients were females. The female to male ratio in our study was 5:1. The mean age at presentation was 35.4 and mean age at malignancy was 35 .

Although solitary thyroid nodules are common in females, they are more likely to be malignant in males $^{9}$. We also noted in our study that male patients are more prone to develop malignancy (60\%).

There are people of different religions with different culture and lifestyles in a junctional place like Siliguri. But incidentally the patients included in my study were from Hindu and Muslim religions. There are also differences in seeking medical attention among the population. In our study we also found that 15 patients (50\%) were in the age group of 20-40 years and $83.3 \%$ patients were females. The female to male ratio in our study was 5:1. The mean age at presentation was 35.4 and mean age at malignancy was 35 .

Although solitary thyroid nodules are common in females, they are more likely to be malignant in males ${ }^{9}$. We also noted in our study that male patients are more prone to develop malignancy $(60 \%)$. like

Geographically this medical college has some specialities

1. It is situated in the sub-Himalayan region which is an area with iodine deficiency.

2. It is the only rural medical college of West Bengal covering largest number of districts.

3. Patients are mostly from backward classes.

4. There are many tea gardens.

Being a route of different destinations there exists massive mixture of populations with different beliefs and cultures. Most of our patients were from Darjeeling and Jalpaiguri districts. $33.33 \%$ patients from Jalpaiguri were found to harbour malignancy. The source of drinking water in most of the cases was well and tube well. Some were dependent upon pond and river water.

We have noted few patients with some other associated clinical features apart from swelling of neck, like hoarseness of voice, fixity to deeper structures and lymph nodal involvement. All the features were associated with malignancy. The size of the nodules does not have any relation with malignancy. A benign colloid nodule may be of huge size where as an just palpable very small swelling may be a case of papillary CA thyroid and even an impalpable papillary CA can present with clinically evident lymph nodes.

The FNAC is a very simple, rapid, easy to perform procedure that is practically free from any complication and radiation exposure. It does not require any anaesthesia and hospitalisation. The procedure is cost effective and inflicts minimal trauma. When this procedure is done under guidance of USG, the sensitivity and accuracy increases. Ultrasound guided FNAC has been found to be an excellent modality for evaluation of those nodules with a non- diagnostic result on conventional (Palpation guided) FNAB. ${ }^{10}$ We also found that USG guided FNAC to be a procedure of simple, easy to do, safe and minimally traumatic procedure that can be performed on OPD basis.

All the patients in our study were operated formulating the operative procedure according to preoperative FNAC results and the specimen were sent for histopathology at college pathology department. All the results were then compared with the preoperative FNAC results as one of the aims of our study was to compare the results of preoperative FNAC with postoperative histopathology report. With this comparison, we have re-evaluated the scope of FNAC in the clinical setting of solitary thyroid nodule and tried to formulate a management plan.

In our study FNAC has correctly diagnosed 26 cases out of 30. The diagnosis of follicular neoplasm on FNAC, whether follicular adenoma or carcinoma has been considered as correct diagnosis as FNAC cannot differentiate between the two. Previous studies as carried out by Chandanwale et al at department of pathology on 150 patients with thyroid nodule showing sensitivity of $90 \%$, specificity of $100 \%$, PPV of $100 \%$ \& NPV of $90 \%$ with diagnostic accuracy of $87.5 \% .{ }^{11}$ Similarly, out of 100 patients evaluated by Md Alamgir Hossain Sikdar sensitivity was $68.75 \%$, specificity $100 \%$ and accuracy was $90 \% .^{12}$ In our study, the sensitivity of the test (FNAC) in detecting malignancy is $75 \%$, specificity is $94.44 \%$ and the accuracy (Effectiveness) of the test is $86.67 \%$. The negative predictive value is $85 \%$ that means $85 \%$ of the patients whose test was negative do not have the disease.

The incidence of malignancy in solitary thyroid nodules varies from $5-20 \%$ in different studies and the incidence is more in the extremes of ages. The likelihood of malignancy increases if the patient is male. Moreover, the risk of malignancy in both solitary nodule and multinodular goitre occurring in endemic goitre areas is reported to be higher than in control areas. ${ }^{13}$ In our study, the overall incidence of malignancy is $40 \%$ (12 cases out of 30 ). In female it is $36 \%$ ( 9 cases out of 25) and in male incidence is $60 \%$ (3 out of 5) which is much higher. The lowest age presenting with malignancy was 18 years. The mean age presenting with malignancy is 35 years.

\section{CONCLUSIONS}

It is evident from our study that USG guided FNAC should be regarded as the investigation of choice in the evaluation of cases with solitary thyroid nodule due to its simplicity, high diagnostic accuracy and absence of significant complications. USG guided FNAC helped in decreasing the extent of surgery by ruling out malignancy. Histopathology was confirmatory and it revealed colloid goitre in most of our benign cases. Papillary carcinoma was most prevalent among the malignancies. Proper counselling and health education are essential for prevention. Early diagnosis, appropriate management and regular follow up are required in minimising suffering of these patients especially in this part of sub-Himalayan region of our country where people are socioeconomically backward. 


\section{REFERENCES}

[1] Robertson ML, Steward DL, Gluckman JL, et al. Continuous laryngeal nerve integrity monitoring during thyroidectomy: does it reduce risk of injury? Otolaryngol Head Neck Surg 2004;131 (5):596-600.

[2] Burch HB, Burman KD, Reed HL, et al. Fine needle aspiration of thyroid nodules. Determinants of insufficiency rate and malignancy yield at thyroidectomy. Acta Cytol 1996;40 (6):1176-83.

[3] Bakhos R, Selvaggi SM, DeJong S, et al. Fine needle aspiration of the thyroid: rate and causes of cytopathologic discordance. Diagn Cytopathol 2000;23 (4):233-7.

[4] Chaplin JM, Sharma N, Watkinson JC. Surgical management of differentiated thyroid cancer. In: Watkinson JC, Gilbert RW, eds. Stell and Maran's textbook of head and neck surgery and oncology. $5^{\text {th }}$ edn. London: Hodder Arnold 2012:421-42.

[5] Bajaj Y, De M, Thompson A. Fine needle aspiration cytology in diagnosis and management of thyroid disease. J Laryngol Oto 2006;120 (6):467-9.

[6] Gupta M, Gupta S, Gupta VB. Correlation of fine needle aspiration cytology with histopathology in the diagnosis of solitary thyroid nodule. J Thyroid Res 2010;2010:1-5.
[7] Morris LF, Ragavendra N, Yeh MW. Evidence based assessment of the role of ultrasonography in the management of benign thyroid nodules. World J Surg 2008;32 (7):1253-63.

[8] Ramchandraiah C, Sadgunachary R, Vijaybhargav. Incidence of malignancy in solitary nodule of thyroid. Indian Journal of Applied Research 2016;6 (2):703-7.

[9] Yeung MJ, Serpell JW. Management of the solitary thyroid nodule. Oncologist 2008;13 (2):105-12.

[10] Braga M, Cavalcanti TC, Collaco LM, et al. Efficacy of ultrasound guided fine-needle aspiration biopsy in the diagnosis of complex thyroid nodules. J Clin Endocrinol Metab 2001;86 (9):4089-91.

[11] Chandanwale S, Singh N, Kumar $H$, et al. Clinicopathological correlation of thyroid nodules. Int J Pharm Biomed Sci 2012;3 (3):97-102.

[12] Sikder MAH, Rahman AZMM, Khair MA. Accuracy of fine needle aspiration cytology (FNAC) in the diagnosis of thyroid swellings. Journal of Dhaka National Med College \& Hospital 2012;18 (2):47-51.

[13] Belfiore A, Giuffrida D, La Rosa GL, et al. High frequency of cancer in cold nodules occurring at young age. Acta Endocrinol (Copenh) 1989;121 (2):197-202. 